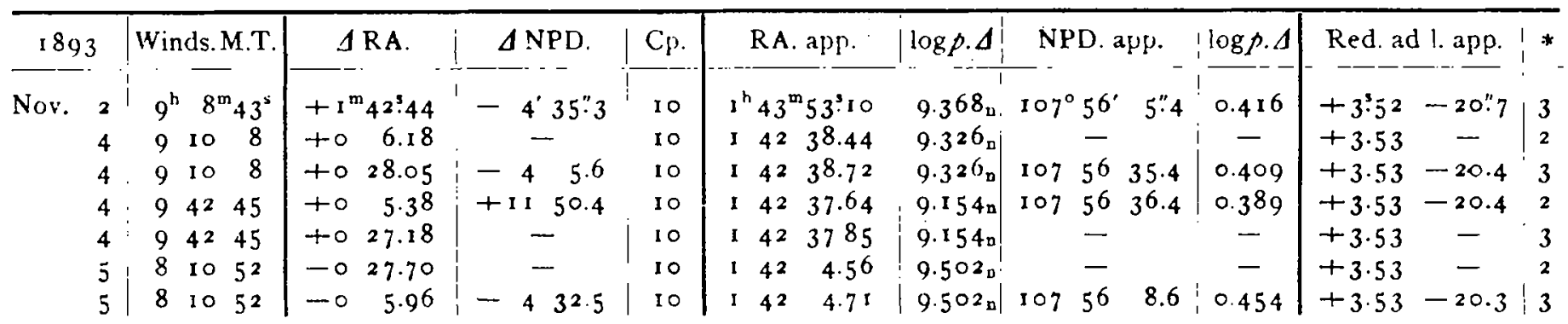

Mean Places of the Comparison Stars for 1893.0.

\begin{tabular}{|c|c|c|c|}
\hline * & RA. & NPD. & Authority \\
\hline I & $I^{h} 50^{m} 45^{s} \cdot 74$ & $107^{\circ} 43^{\prime} 50^{\prime \prime} .5$ & $\mathrm{AOe}_{2}$ I $\mathrm{I} 68$ \\
\hline 2 & $142 \quad 28.73$ & $10745 \quad 6.4$ & Lal. $3294, \mathrm{AOe}_{2}$ × 868 \\
\hline 3 & 1427.14 & $108 \quad I$ & I.al. $3288, \mathrm{AOe}_{2}$ I $066, \mathrm{Y}_{3} 86 \mathrm{x}$ \\
\hline
\end{tabular}

Private Observatory, The Peninsula, Windsor N. S. Wales, 1893 Dec. 1.

Fohn Tebbutt.

\title{
Scbreiben von Herrn Prof. L. Weinek, Director der k. k. Sternwarte in Prag, betr. die Prager Beobachtungen des Cometen von 1811.
}

In Bezug auf die Erklärung, die Dr. Herz dafur giebt, dass er in seiner "Bestimmung der Bahn des Cumeten von 1811* die Prager Beobachtungen nicht benutzt hat (vgl. Publicationen der v. Kuffner'schen Sternwarte, Bd. II, S. 52 und 175), erlaube ich mir hier auszusprechen, dass Dr. Herz von der ihm angebotenen Erlaubniss, die betreffenden Manuscripte der Prager Sternwarte auf der k. k. Wiener Sternwarte einzusehen und zu benutzen, nicht Gebrauch zu machen fur gut fand, und dass mich somit keine Schuld trifft, wenn die genannten Beobachtungen nicht verwendet wurden. Ich bin erst jetzt durch die Anzeige in der V.J.S. d. Astr. Ges. 28. Jahrg. S. 267 auf die Herz'sche Arbeit, die mir bisher unbekannt geblieben war, gefuhrt worden.

Prag, k. k. Sternwarte, I 894 Febr. 13.

I. Weinek.

\section{Entdeckung von drei neuen Planeten $1894 \mathrm{AX}, \mathrm{AY}$ und $\mathrm{AZ}$.}

Telegramm aus Berlin. Zwei Planeten Wolf I. März:
$1894 \Delta X$
$\mathrm{AR} .=166^{\circ} 9^{\prime}$
PD. $=83^{\circ} \mathbf{I}^{\circ}$
$=855^{2}$
tägl. Bew. $-19^{\circ} \quad 0^{\circ} \quad$ Gr. $8^{\mathrm{m}}$
$1894 \Delta Y, 940.0 \geqslant>\quad=16830$
$>\quad-12^{\circ}-8^{\circ}$
- $12^{\mathrm{m}}$ Berberich.

Nach ciner Mittheilung von Herrn A. Berberich d. d. 4. März ist der Planet 1894 AX an 3 . März von Herrn G. Witt auf der Urania-Sternwarte beobachtet worden; die Grösse des Planeten ist darnach höchstens $9 \cdot 5$ oder 9.6 .

Telegramm aus Paris. Planète Courty 5 Mars:

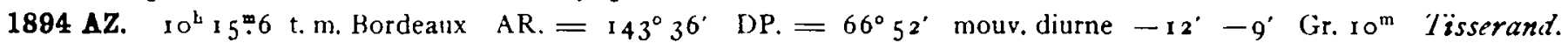

\section{Literarische Anzeige.}

C. Easton. La voie lactée dans l'hémisphère boréal. Dordrecht, Blussé et Co. Paris, Gauthier-Villars. Einem empfehlenden Vorwort von Prof. H. G. van de Sande-Bakhuyzen folgt eine Finleitung (S. 7-1 4), dann historische Notizen (S. 15-37) und hierauf Beschreibungen der einzelnen Theile der nördlichen Milchstrasse nebst Angaben der Coordinaten. Fünf von dem Verfasser selbst lithographirte und zum Theil eigenhändig retouchirte Karten geben ein sehr fein gehaltenes Rild der Milchstrasse.

Kr.

Inhalt zu Nr. 3217. H. Kayser. Ueber den Finfluss der Spaltweite auf das Aussehen der Cometenspectra. I. -- L. Steiner. Aufforderung betr. Beubaclitungen des Cometen $1892 \mathrm{II}$. 9. - E. v. Cothard. Der kleine Barnard'sche Nebel bei M. 57. Ir. - M. Wolf. Zur Erkläiung der liettenbildung der (icstine. 11. - D. Peyra. Sopra una possibile connessione della cometa i893 IV colle comete 1864 I e 1822 I. 1 3. - F. Te6butt. Observations of Minor Planet (6) Hebe at Windsor. N. S. Wales. 13. - Schreiben von Herm Prof. L. Weinek, Director der k. k. Sternwarte in Prag. 15. - Entdeckung von diei neuen Planeten I894 AX, AY und AL. 15. - Literarische Anseige. 15

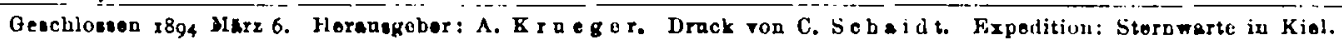

